\title{
Functional and Aesthetic Reconstruction of Shoulder Using Pedicled LD Flap after Radical Debridement of Tuberculosis Infected Rheumatoid Arthritis Wound
}

\author{
Jeongseok Oh ${ }^{1}$, Hee Chang Ahn ${ }^{1 *}$, Bong Gun Lee ${ }^{2}$ \\ ${ }^{1}$ Department of Plastic \& Reconstructive Surgery, Hanyang University Medical Center, Seoul; ${ }^{2}$ Department of Orthopedic Surgery, Hanyang University Medical \\ Center, Seoul, Korea
}

\begin{abstract}
Reconstruction of large shoulder defects is challenging, especially in complicated chronic wounds. We report a case of a tuberculosis-infected shoulder defect in a patient with rheumatoid arthritis (RA) that was successfully reconstructed with pedicled latissimus dorsi (LD) musculocutaneous flap coverage. A 57-year-old male visited our department with a severely painful open wound $(0.5 \mathrm{~cm} \times 0.5 \mathrm{~cm})$, later confirmed to be tuberculosis (TB) infected, in the right anterior shoulder region. Several surgical interventions including open synovectomy had been performed at a local orthopedic clinic before the patient presented at our institute. We performed radical debridement and successfully reconstructed the defect with a combination of TB medication, corticosteriod tapering, complete removal of non-viable tissue, and a pedicled LD flap to provide adequate skin and soft tissue volume. Chronic shoulder wounds should be carefully examined for TB infections, which, although extremely rare, can lead to joint destruction and extensive soft tissue defects when diagnosis is delayed. In terms of flap choice, the pedicled LD musculocutaneous flap has many advantages for functional and aesthetic reconstruction of a large defect in the shoulder region.
\end{abstract}

Keywords: Surgical flaps, Wound healing, Reconstructive surgical procedures

\section{Introduction}

Shoulder reconstruction is a delicate task involving complex joint structure and unique function [1]. Microsurgical advancements have provided high success rates for various flap techniques and offer many options for successful achievement of the primary goal, replacement of similar tissue [2]. The goal of such methods has shifted to more efficient or optimal flap selection, thereby minimizing the low, but not negligible failure rate, or other complications [3].

Shoulder defects can be caused by sarcoma resection, trauma, and chronic wounds [4]. Associated comorbid conditions may lead to sub-optimal wound healing [5]. Radical debridement or wide resection is often needed for treatment, but yields large defects. Hence, adequate soft tissue coverage is crucial for aesthetic reasons and functional recovery $[1,2]$.

Pedicled latissimus dorsi (LD) flaps are popular for the thin, pliable skin paddle with a thin, large fan-like muscle component with a constant long pedicle [1]. This allows a wide range of versatility in reconstructing complex defects, even when accounting for unexpected changes of defect during operation. We present a rare manifestation of shoulder joint tuberculosis infection over rheumatoid arthritis (RA), which eventual- \\ Case Report \\ Received: June 29, 2018 \\ Revised: July 31, 2018 \\ Accepted: August 1, 2018

\section{Corresponding author:} \\ Hee Chang Ahn, M.D. \\ Department of Plastic \& Reconstructive Surgery, \\ Hanyang University Medical Center, 222-1 \\ Wangsimni-ro, Seongdong-gu, Seoul 04763, Korea \\ Tel: +82-2-2295-8560 \\ Fax: +82-2-2295-7671 \\ E-mail: ahnhc@hanyang.ac.kr
}

This is an Open Access article distributed under the terms of the Creative Commons Attribution Non-Commercial License (http://creativecommons.org/licenses/by-nc/4.0/) which permits unrestricted non-commercial use, distribution, and reproduction in any medium, provided the original work is properly cited.

(c) 2018 Korean Wound Management Society 
ly led to the diagnosis of pulmonary TB that was successfully cured and reconstructed with pedicled LD musculocutaneous flap coverage.

\section{Case}

A 57-year-old man was transferred to our unit with right shoulder pain and exudate from a previous surgical incision site (Fig. 1). Exudate was evident in the small open wound (size 0.5 $\mathrm{cm} \times 0.5 \mathrm{~cm}$ ), at the distal end of the $11-\mathrm{cm}$-long previous incision site. The patient was not able to move his shoulder due to severe pain. Before visiting us, the patient had been diagnosed as a hepatitis B virus carrier with RA four years prior and took oral corticosteroid medications (oral prednisolone 5-15 mg daily) along with several local corticosteroid injections for shoulder joint pain. Shoulder pain was well controlled until acute aggravation 13 months prior; as a result, the patient had undergone open synovectomy the following month. Humerus head resection with vancomycin mixed cement bead insertion (Fig. 2) was performed, as osteomyelitis was observed via magnetic resonance imaging (MRI). Wound culture did not reveal any bacteria. While not able to determine the exact cause of the non-healing wound and shoulder pain, the local orthopedic clinic irrigated the wound several times and tried several different doses of oral prednisolone in an attempt to control the suspected RA flare up.

In the patient's first visit to our center, it was noted to be odd that a rather small wound with several medical and surgical treatments all failed to accomplish wound healing for several years, while conventional wound culture tests did not reveal any infectious organisms. These symptoms suggested a chron-

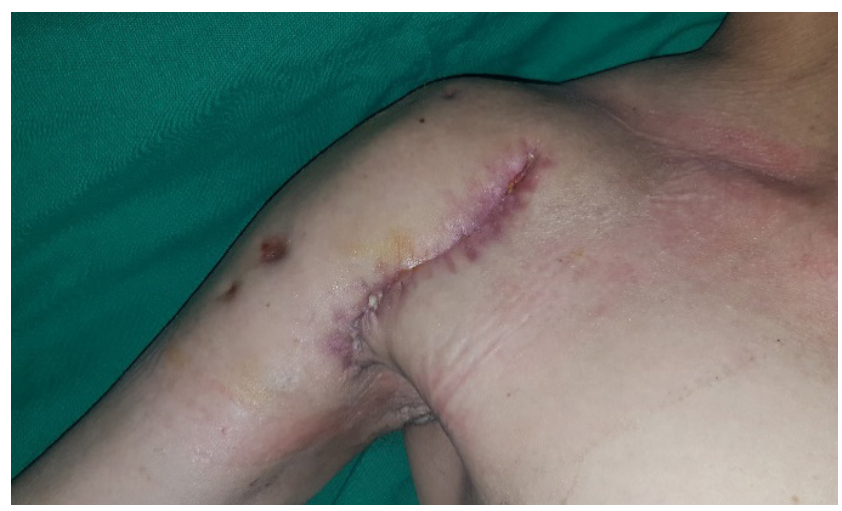

Fig. 1. Gross preoperative photograph of the chronic wound on the right shoulder. Previous incisional scar (length) is observed. The distal open wound $(0.5 \mathrm{~cm} \times 0.5 \mathrm{~cm})$ was not healed with evident exudative discharge. ic wound with the cause not yet clearly revealed. Hence, we performed tests for other causes like fungal disease and mycobacterium.

The rapid acid-fast bacteria (AFB)-staining (Ziehl Neelsen) tests showed positive results. Upon consultation with both the pulmonary and infectious disease departments, TB medication (isoniazid, ethambutol, rifampicin, pyrazinamide) was started immediately, as pulmonary TB was revealed by chest computed tomography (CT) imaging study. Upon consultation with the rheumatology department, oral prednisolone was tapered to $2.5 \mathrm{mg}$ daily. Mycobacterium tuberculosis was confirmed with tuberculosis culture tests in the sputum and discharge of the shoulder wound.

The shoulder wound was managed with conservative dressings until the patient was prepared for general anesthesia. Contracture and an extensive amount of non-viable tissue required preoperative planning of radical debridement from the orthopedic department and a reconstruction method that provided an abundant soft tissue volume and large skin paddle. We designed an ipsilateral pedicled LD musculocutaneous flap with a skin paddle that includes perforators of the vertical branch of

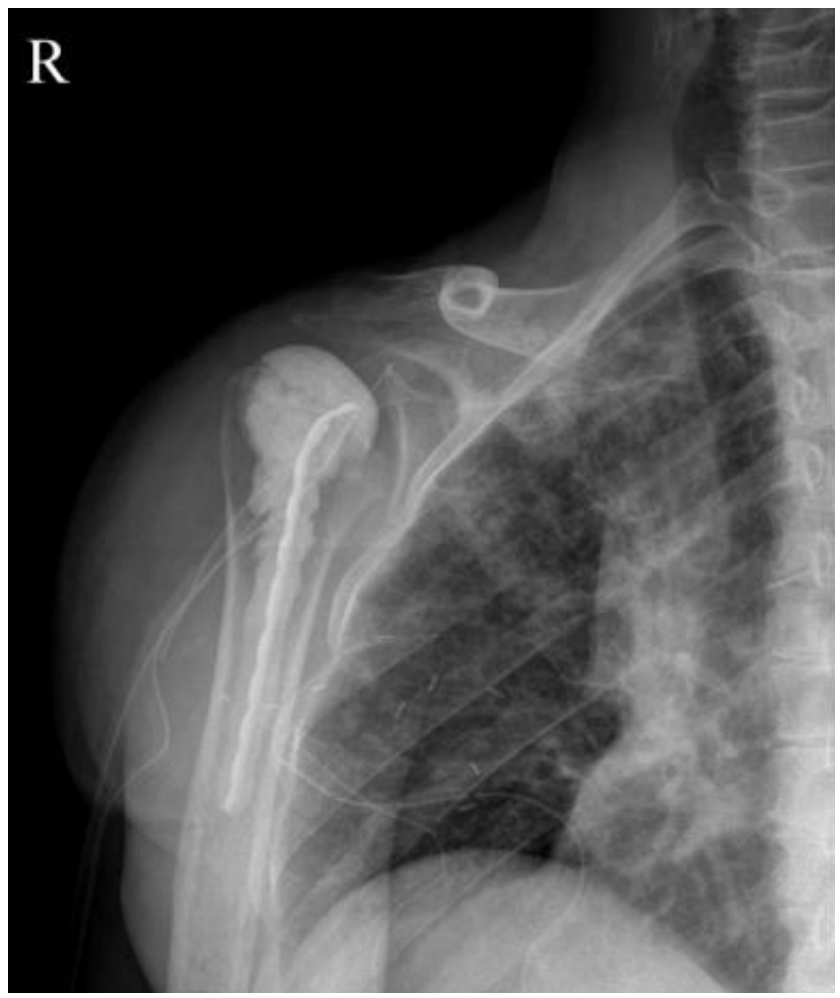

Fig. 2. Preoperative x-ray of the right shoulder. Synovectomy, humerus head resection, bone cement \& vancomycin bead insertion was performed in the previous local orthopedic clinic. 
the thoracodorsal artery. The orthopedic team removed the previously inserted bone cement, performed radical debridement of unviable tissue, irrigated the wound, sent the specimen for biopsy and wound culture studies (later confirmed to be free from malignancy and bacteria), and then re-inserted amikacin-mixed cement with a 1/3 tubular plate. (Fig. 3) Our

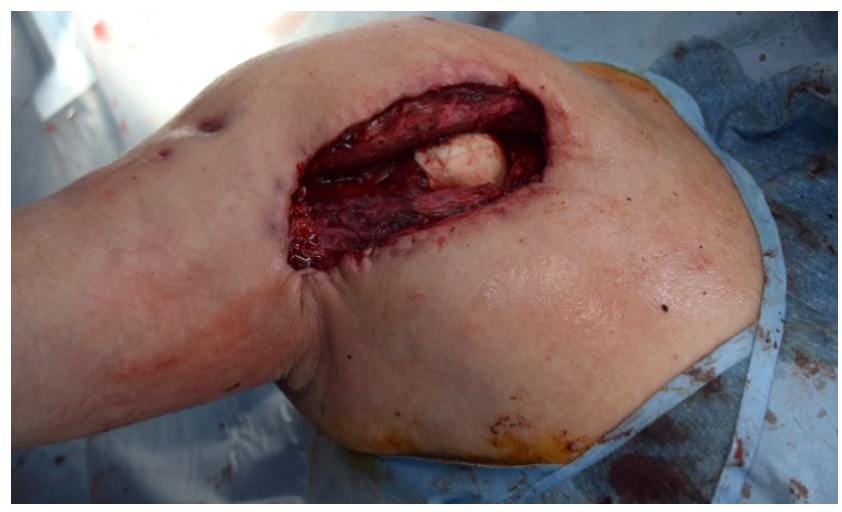

Fig. 3. Intraoperative photograph of the right shoulder. Previously inserted bone cement, vancomycin bead was removed. Radical debridement of non-viable tissue and amikacin-mixed cement was inserted by the orthopedic department. The final defect size was $11 \mathrm{~cm} \times 5 \mathrm{~cm}$.

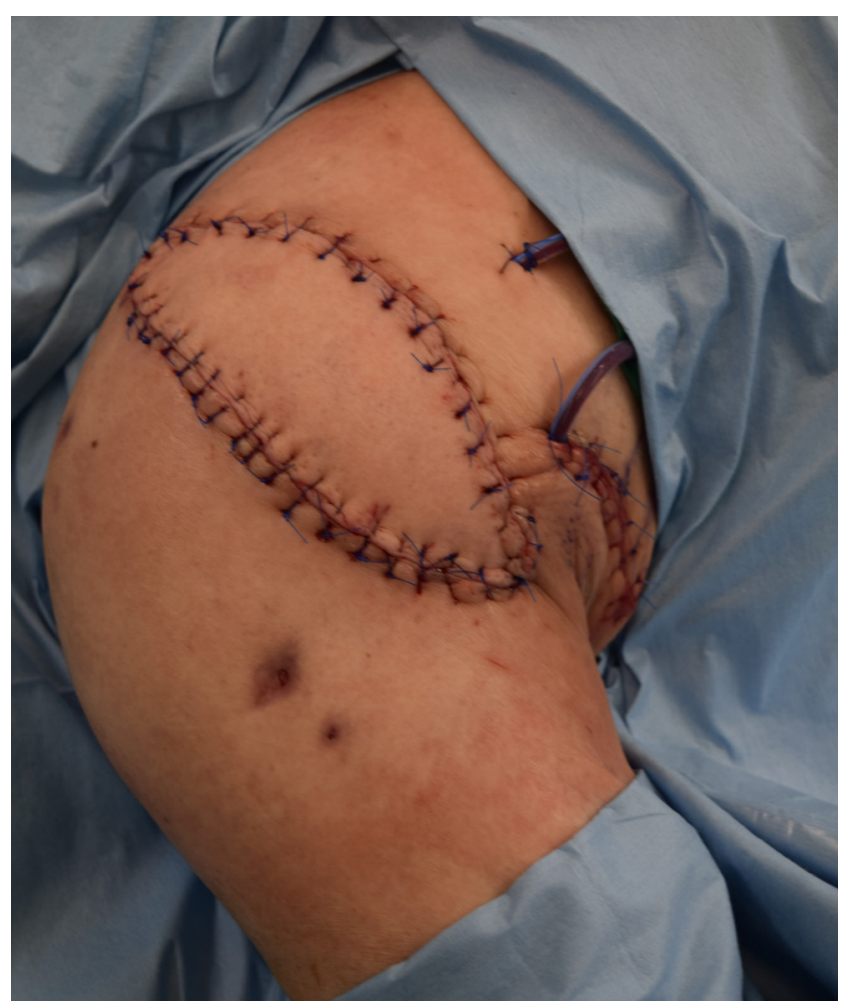

Fig. 4. Immediate postoperative photograph. $11 \mathrm{~cm} \times 5 \mathrm{~cm}$ sized ipsilateral pedicled LD musculocutaneous flap was transferred to the defect to restore the convex structure. team evaluated the defect and harvested the ipsilateral pedicled LD musculocutaneous flap with a $11 \mathrm{~cm} \times 5 \mathrm{~cm}$ skin paddle and adequate muscle volume for dead space obliteration. The flap was successfully transferred to the defect (Fig. 4), and donor site was primarily closed. The patient was discharged in three months without any wound complications. After one

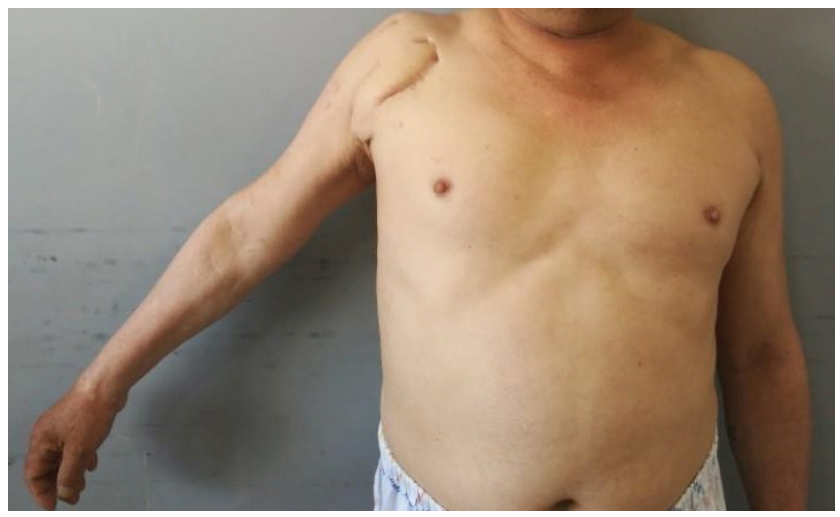

Fig. 5. 1 year postoperative photograph. Both recipient and donor site is completely healed. Shoulder abduction of 45 degrees is available without any discomfort or pain.

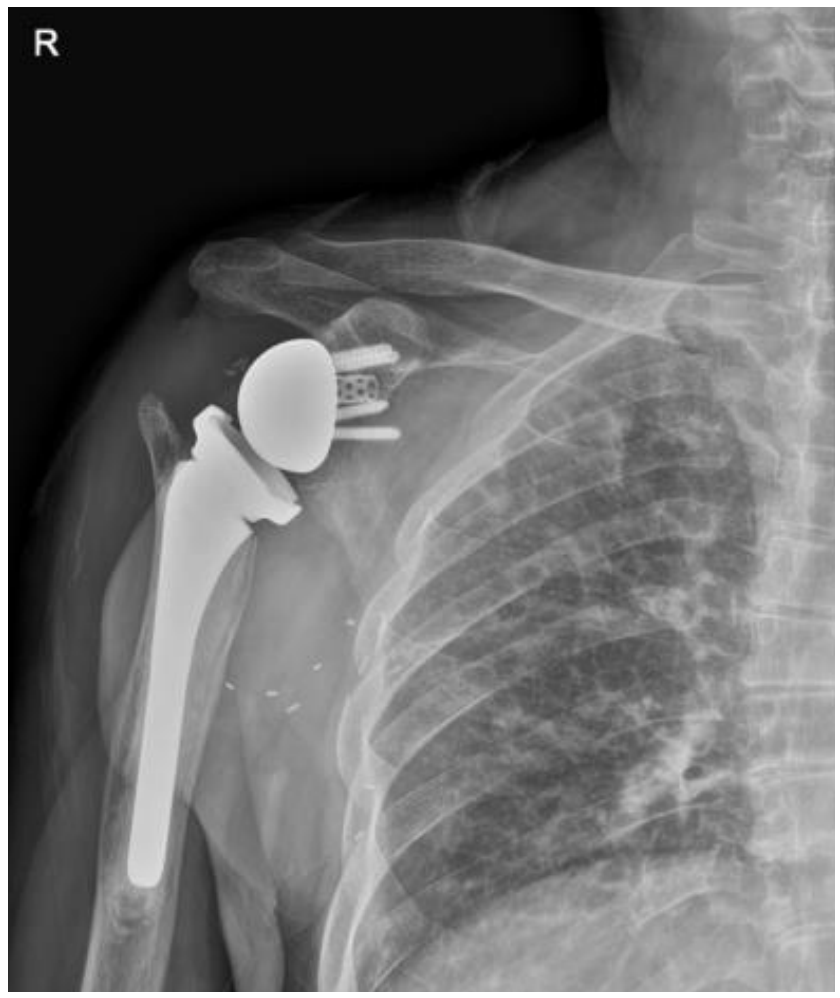

Fig. 6. X-ray of right shoulder after arthroplasty was performed. 
year, arthroplasty was performed for further recovery of shoulder function (Figs. 5, 6).

\section{Discussion}

The shoulder is the junction between the trunk and arm, consisting of a complex joint, muscles, and soft tissue. Shoulder reconstruction is challenging due to the convex structure that controls one of the most important joints in humans, with the widest range of motion $[1,2]$.

Shoulder defects can be caused by various factors. Regardless of the etiology, sufficient debridement or excision before reconstruction is key for optimal wound healing. Immunosuppressive drugs for arthritis and radiotherapy for sarcoma are well-known factors for unfavorable wound healing [6,7]. Compromised debridement puts local recurrence or incomplete malignancy removal at risk for sarcoma, biofilms in chronic wounds, or irreversible joint destruction in chronic infection wounds $[1,8]$. The challenge lies in the balance of complete evacuation vs. detrimental destruction of joint structure. RA, TB, and wound infection combined can increase treatment complexity. In an attempt to control RA flare up with corticosteroids, wound healing can be negatively impacted. Occasionally, defects can unexpectedly be larger than expected. Provided high reliability of a reconstructive option, a surgeon's reluctance toward proper debridement can be minimized.

Primary closure of wounds or coverage with inadequate soft tissue can cause contracture and restrict shoulder motion, which can be detrimental to the patient's quality of life. Various reconstructive options can be considered depending on the extent of defect. Primary closure can be limitedly performed in cases with no vital structure exposure and tension free closures [1]. Sufficiently undermined adjacent tissue can be sufficient for closure, but scar tissue can restrict motion.

For large defects, latissimus dorsi and pectoralis major muscles can be used as pedicled flaps with adequate tissue volume, while eliminating the rare, but not negligible, risks of microsurgical free flaps. The muscle volume present in these muscle groups is favorable for restoring the convex contour [3]. Since the first introduction of pedicled LD flaps by Tansini in 1906, it has been considered a solid option for upper extremity reconstruction due to the abundant vascular supply, constant anatomy, and adequate soft tissue volume [1,2].

Shoulder defects often involve exposure of implants or bones. Postoperative infections can be deep, resulting in joint destruc- tion. Well vascularized muscle facilitates wound healing and reduces infection risk $[1,9]$.

Tuberculosis is a very rare disease in industrialized countries. Musculoskeletal TB represents approximately 10 to 35\% of extra-pulmonary cases. Shoulder joint incidence represents 1 to $2.8 \%$ of skeletal tuberculosis, after spine, hip, and knee $[10,11]$. Uncommon inflammatory conditions like this are prone to delayed diagnosis, because shoulder pain is commonly approached from a mechanical origin of rotator cuff pathology [10]. The slow, insidious, and dry behavior with a variety of clinical, pathological, and radiological features makes the diagnosis even more difficult [11].

The delayed diagnosis accounts for the large extent of questionable viability and subclinical infection of the surrounding tissue. As a result, shoulder joint tuberculosis can mimic sarcoma, resulting in large defects of soft tissue and vital structures [12]. Adequate soft tissue coverage is crucial for functional and structural restoration of the unique shoulder joint. As tuberculosis is often observed as an opportunistic infection in immunosuppressed patients, wound healing is sub-optimal [5, 13]. These unfavorable conditions complicate shoulder reconstruction involving tuberculosis infection.

Unsuccessful wound healing with conventional treatment methods may suggest a chronic wound with other causes like fungus, opportunistic infections, or even tuberculosis. Suspecting various diseases is important for accurate diagnosis and treatment. In our case, shoulder motion could be maintained with proper diagnosis, appropriate medical management, radical debridement, and flap coverage.

Our reconstruction of a large, extensive shoulder defect with pedicled LD flap was achieved with a multi-disciplinary approach to manage the combined TB infection, underlying RA, and operative planning with the orthopedic team. Versatility of the free thoracodorsal artery flap was obtained with minimal microsurgical skills. This method can be useful for virtually any defect without altering the extent of debridement or encountering an unexpectedly large defect during surgery.

\section{References}

1. Behnam AB, Chen CM, Pusic AL, et al. The pedicled latissimus dorsi flap for shoulder reconstruction after sarcoma resection. Ann Surg Oncol 2007;14:1591-5. Epub 2007 Feb 10.

2. Lohman RF, Nabawi AS, Reece GP, et al. Soft tissue sarcoma of the upper extremity: a 5-year experience at two insti- 
tutions emphasizing the role of soft tissue flap reconstruction. Cancer 2002;94:2256-64.

3. D'Aniello C, Grimaldi L, Bosi B, et al. Shoulder reconstruction by latissimus dorsi myocutaneous flap based on the serratus branch after advanced soft-tissue sarcoma excision. Plast Reconstr Surg 2000;105:2082-5.

4. Ma CH, Tu YK, Wu CH, et al. Reconstruction of upper extremity large soft-tissue defects using pedicled latissimus dorsi muscle flaps--technique illustration and clinical outcomes. Injury 2008;39 Suppl 4:67-74

5. Paul WA, David AH. Influence of comorbidities: neuropathy, vasculopathy, and diabetes on healing response quality. Adv Wound Care (New Rochelle) 2013;2:410-21.

6. Sbitany H, Xu X, Hansen SL, et al. The effects of immunosuppressive medications on outcomes in microvascular free tissue transfer. Plast Reconstr Surg 2014;133:552e-8e.

7. Frank $\mathrm{H}$, Elisabeth $\mathrm{O}$, Fabian $\mathrm{P}$, et al. Wound healing after radiation therapy: review of the literature. Radiat Oncol 2012;7:162.

8. McCarty SM, Cochrane CA, Clegg PD, et al. The role of endogenous and exogenous enzymes in chronic wounds: a fo- cus on the implications of aberrant levels of both host and bacterial proteases in wound healing. Wound Repair Regen 2012;20:125-36.

9. Shaw WW, and Hidalgo DA. General concepts of free tissue transfer in trauma. In Microsurgery in Trauma. Mt. kisco, N.Y.: Futura, 1987.

10. Monach PA, Daily JP, Rodriguez-Herrera G. Tuberculous osteomyelitis presenting as shoulder pain. J Rheumatol 2003; 30:851-6.

11. Mangwani J, Gupta AK, Yadav CS, et al. Unusual presentation of shoulder joint tuberculosis: a case report. J Orthop Surg (Hong Kong) 2001;9:57-60.

12. Altaf Mir M, Ahmad I, Yaseen M. Primary Tuberculosis of Hand Soft Tissue. World J Plast Surg 2016;5:313-8.

13. Rutherford Al, Patarata E, Subesinghe S, et al. Opportunistic infections in rheumatoid arthritis patients exposed to biologic therapy: results from the British Society for Rheumatology Biologics Register for Rheumatoid Arthritis. Rheumatology (Oxford) 2018;57:997-1001. doi: 10.1093/rheumatology/ key023. 